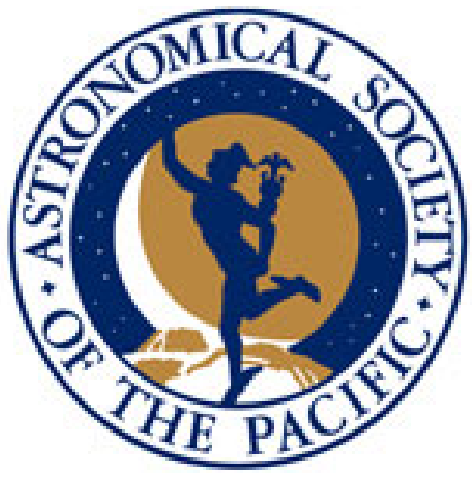

Grids of ATLAS9 Model Atmospheres and MOOG Synthetic Spectra

Author(s): Evan N. Kirby

Source: Publications of the Astronomical Society of the Pacific, Vol. 123, No. 903 (May 2011), pp. 531-535

Published by: The University of Chicago Press on behalf of the Astronomical Society of the Pacific

Stable URL: http://www.jstor.org/stable/10.1086/660019

Accessed: 10/06/2011 10:50

Your use of the JSTOR archive indicates your acceptance of JSTOR's Terms and Conditions of Use, available at http://www.jstor.org/page/info/about/policies/terms.jsp. JSTOR's Terms and Conditions of Use provides, in part, that unless you have obtained prior permission, you may not download an entire issue of a journal or multiple copies of articles, and you may use content in the JSTOR archive only for your personal, non-commercial use.

Please contact the publisher regarding any further use of this work. Publisher contact information may be obtained at http://www.jstor.org/action/showPublisher?publisherCode=ucpress.

Each copy of any part of a JSTOR transmission must contain the same copyright notice that appears on the screen or printed page of such transmission.

JSTOR is a not-for-profit service that helps scholars, researchers, and students discover, use, and build upon a wide range of content in a trusted digital archive. We use information technology and tools to increase productivity and facilitate new forms of scholarship. For more information about JSTOR, please contact support@jstor.org.

The University of Chicago Press and Astronomical Society of the Pacific are collaborating with JSTOR to digitize, preserve and extend access to Publications of the Astronomical Society of the Pacific. 
Publications of the Astronomical Society of the Pacific, 123:531-535, 2011 May

(C) 2011. The Astronomical Society of the Pacific. All rights reserved. Printed in U.S.A.

\title{
Grids of ATLAS9 Model Atmospheres and MOOG Synthetic Spectra
}

\author{
EVAN N. KIRBY ${ }^{1,2}$ \\ Received 2011 February 14; accepted 2011 March 5; published 2011 April 5
}

\begin{abstract}
A grid of ATLAS9 model atmospheres has been computed, spanning $3500 \mathrm{~K} \leq T_{\text {eff }} \leq 8000 \mathrm{~K}$, $0.0 \leq \log g \leq 5.0,-4.0 \leq[\mathrm{M} / \mathrm{H}] \leq 0.0$, and $-0.8 \leq[\alpha / \mathrm{Fe}] \leq+1.2$. These parameters are appropriate for old stars in the red giant branch, subgiant branch, and the lower main sequence. The main difference from a previous similar grid is the range of $[\alpha / \mathrm{Fe}]$ values. A grid of synthetic spectra, calculated from the model atmospheres, is also presented. The fluxes are computed every $0.02 \AA$ from $6300 \AA$ to $9100 \AA$. The microturbulent velocity is given by a relation to the surface gravity. This relation is appropriate for red giants, but not for subgiants or dwarfs. Therefore, caution is urged for the synthetic spectra with $\log g>3.5$ or for any star that is not a red giant. Both the model atmosphere and synthetic spectrum grids are available online through VizieR. Applications of these grids include abundance analysis for large samples of stellar spectra and constructing composite spectra for stellar populations.
\end{abstract}

\section{INTRODUCTION}

One approach to measuring the composition of a star from low- or medium-resolution spectroscopy is to synthesize the stellar spectrum. An essential ingredient in the synthesis is a model atmosphere. Typically, a model atmosphere tabulates quantities such as density, temperature, pressure, electron number density, and opacity as a function of optical depth. A grid of synthetic spectra at a range of atmospheric parameters (effective temperature, surface gravity, and composition) streamlines the measurement of these parameters from the observed spectrum. Therefore, it is useful to have a grid of model atmospheres, from which a grid of synthetic spectra may be computed.

Mihalas (1965) and Strom \& Avrett (1965) generated the first grids of nongray, continuum model atmospheres. The use of opacity distribution functions (ODFs; Strom \& Kurucz 1966) greatly simplifies the computation of such grids. ODFs treat the absorption coefficient as smoothly varying within wavelength subdivisions of the spectrum. In effect, the information about line opacity is compressed into a compact function, which may be used to compute model atmospheres.

The grid of model atmospheres most relevant to the current work is that of Castelli \& Kurucz (2003). They computed a grid of model atmospheres with the ATLAS9 program (Kurucz 1993a). Kurucz (1970) described the original ATLAS code in great detail. Castelli \& Kurucz (2003) sampled a wide range of effective temperatures ( $\left.T_{\text {eff }}\right)$, surface gravities $(\log g)$, and metallicity $([\mathrm{M} / \mathrm{H}])$. They also sampled two values of $[\alpha / \mathrm{Fe}]$ : 0.0 and +0.4 .

\footnotetext{
${ }^{1}$ Hubble Fellow.

${ }^{2}$ California Institute of Technology, 1200 East California Boulevard, Mail Code 249-17, Pasadena, CA 91125.
}

One major use of stellar atmospheres is the computation of synthetic stellar spectra for the purpose of measuring chemical abundances. Because it is computationally expensive to generate a model atmosphere, it is sometimes wise to invest the computational resources up front by generating a grid of model atmospheres. Synthetic spectra can then be computed from atmospheres interpolated within the grid. The red to near-infrared spectral region is the subject of this article. There is a precedent for synthetic spectral grids in this spectral region (e.g., Coelho et al. 2005; Munari et al. 2005; Palacios et al. 2010). The synthetic spectral grid here is unique because of its wide range in the $\alpha$ enhancement $([\alpha / \mathrm{Fe}])$.

Kirby et al. (2010) measured the detailed abundances of thousands of red giant stars in the dwarf satellite galaxies of the Milky Way. In order to do so, they required a grid of synthetic spectra. A grid of model atmospheres was computed at a range of $[\alpha / \mathrm{Fe}]$ so that the compositions of the atmospheres would be consistent with the spectra. The inspiration for this procedure was the grid of Castelli \& Kurucz (2003). However, the presence in dwarf galaxies of stars with large $[\alpha / \mathrm{Fe}]$ (Frebel et al. 2010) and small $[\alpha / \mathrm{Fe}]$ (Letarte et al. 2010) required a broader range of $[\alpha / \mathrm{Fe}]$ in the model atmospheres. Therefore, Castelli \& Kurucz's (2003) computation of model atmospheres was repeated, but for a wide range in $[\alpha / \mathrm{Fe}]$. A grid of synthetic spectra was computed based on the grid of model atmospheres.

The purpose of this article is to make these grids publicly available. Those with possible interests in these grids include researchers who want to measure the elemental abundances for a large number of far-red spectra or who want to generate composite spectra of stellar populations for an integrated light analysis. Other uses are also encouraged.

Table 1 gives the details of the grid parameters. The step size for $[\mathrm{M} / \mathrm{H}]$ is smaller for the spectra than for the atmospheres 
TABLE 1

ATMOSPHERIC PARAMETER GRID

\begin{tabular}{|c|c|c|c|c|c|}
\hline Quantity & Minimum & Maximum & & Step & Number \\
\hline$T_{\text {eff }}(\mathrm{K})$ & 3500 & 8000 & $\left\{\begin{array}{l}100 \\
200\end{array}\right.$ & $\begin{array}{l}T_{\text {eff }} \leq 5500 \\
T_{\text {eff }} \geq 5600\end{array}$ & 34 \\
\hline $\log g\left(\mathrm{~cm} \mathrm{~s}^{-2}\right), T_{\text {eff }} \leq 6800$ & 0.0 & 5.0 & & 0.5 & 11 \\
\hline $\log g\left(\mathrm{~cm} \mathrm{~s}^{-2}\right), T_{\text {eff }} \geq 7000$ & 0.5 & 5.0 & & 0.5 & 10 \\
\hline$[\mathrm{M} / \mathrm{H}]($ atmospheres) $\quad \ldots \ldots \ldots \ldots \ldots \ldots \ldots$ & -4.0 & 0.0 & & 0.5 & 9 \\
\hline 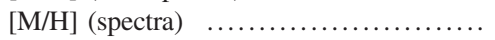 & -4.0 & 0.0 & & 0.1 & 41 \\
\hline 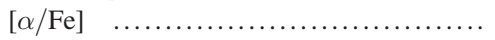 & -0.8 & +1.2 & & 0.1 & 21 \\
\hline
\end{tabular}

NOTE.-Sections 2 and 3 give the parameterization of microturbulent velocity, $\xi$.

(see $\S 3$ ). Section 2 describes the generation of the grid of model atmospheres, and $\S 3$ describes the grid of synthetic spectra. Section 4 explains how the data may be retrieved from the VizieR online catalog.

\section{ATLAS9 MODEL ATMOSPHERES}

The grid (Table 1) of plane-parallel model atmospheres in local thermodynamic equilibrium (LTE) was computed with Kurucz's (1993a) DFSYNTHE, KAPPA9, and ATLAS9 codes ported into Linux (Sbordone et al. 2004; Sbordone 2005) and compiled with the Intel FORTRAN compiler 11.1. All computations were performed in serial on 256 processors at the University of California, Santa Cruz, Pleiades Linux computing cluster. The compositions of the ODFs were the scaled solar abundances of Anders \& Grevesse (1989) except that the abundance of iron was $12+\log \epsilon(\mathrm{Fe})=7.52 .{ }^{3}$ The $\alpha$ elements $\mathrm{O}$, $\mathrm{Ne}, \mathrm{Mg}, \mathrm{Si}, \mathrm{S}, \mathrm{Ar}, \mathrm{Ca}$, and $\mathrm{Ti}$ were additionally scaled by the parameter $[\alpha / \mathrm{Fe}]$.

The starting point for an ATLAS9 model atmosphere is an ODF. ODFs were computed in a manner identical to Castelli \& Kurucz (2003) except for the elemental composition. In summary, DFSYNTHE (described by Castelli 2005) was used to generate an ODF at each point in the stellar parameter grid. F. Castelli's scripts ${ }^{4}$ were employed for this purpose. These scripts compute ODFs at lower and higher effective temperatures than the grid presented here, but keeping the file formats consistent with Castelli's scripts simplified later steps in computing the model atmospheres. The lists of atomic and some molecular (including $\mathrm{H}_{2}, \mathrm{HD}, \mathrm{CH}, \mathrm{C}_{2}, \mathrm{CN}, \mathrm{CO}, \mathrm{NH}, \mathrm{OH}$, $\mathrm{MgH}, \mathrm{SiH}$, and $\mathrm{SiO}$, but not including $\mathrm{TiO}$ or $\mathrm{H}_{2} \mathrm{O}$ ) transitions, were those of R. L. Kurucz. ${ }^{5}$ See Kurucz (1990) for details of

\footnotetext{
${ }^{3}$ The symbol $\epsilon(X)$ is the ratio of the number density $(n)$ of atoms of element $X$ to the number density of hydrogen atoms: $\epsilon(X)=n(X) / n(\mathrm{H})$. However, note that the ATLAS code accepts abundances expressed as $n(X) / n_{\text {total }}$. This work assumes the solar helium-to-hydrogen number ratio of 0.098 (Anders \& Grevesse 1989). Also note that the solar abundance pattern assumed here (Anders \& Grevesse 1989) is different from the solar abundance pattern of Grevesse \& Sauval (1998), which is the default for the DFSYNTHE and ATLAS9 codes.

${ }^{4}$ See http://wwwuser.oat.ts.astro.it/castelli/.

${ }^{5}$ Downloaded from http://wwwuser.oat.ts.astro.it/castelli/sources/dfsynthe .html.
}

the line lists. The molecular line lists for $\mathrm{TiO}$ and $\mathrm{H}_{2} \mathrm{O}$ were provided by Schwenke (1998) and Partridge \& Schwenke (1997), respectively. The KAPPA9 code in the same software package as DFSYNTHE computed Rosseland mean opacities from the ODFs.

For consistency with Castelli \& Kurucz's (2003) grid of ATLAS9 atmospheres, convective overshooting was turned off, and the mixing length parameter for convection was $l / H_{p}=$ 1.25. For each combination of $T_{\text {eff }}, \log g,[\mathrm{M} / \mathrm{H}]$, and $[\alpha / \mathrm{Fe}]$, there are two atmospheres with two different values of microturbulent velocity, $\xi$. The values are the two velocities among $0,1,2$, and $4 \mathrm{~km} \mathrm{~s}^{-1}$ that bracket the microturbulent velocity appropriate for the star's surface gravity (equ. 2 of Kirby et al. 2009): $\xi\left(\mathrm{km} \mathrm{s}^{-1}\right)=2.13-0.23 \log g$.

ATLAS9 requires an input model atmosphere as an initial guess. An ATLAS9 model atmosphere with similar $T_{\text {eff }}, \log g$, and $[\mathrm{M} / \mathrm{H}]$ from Castelli \& Kurucz's (2003) grid was used for this purpose. At least 30 iterations were computed for each stellar atmosphere. After 30 iterations, convergence was tested by examining the difference in the flux and flux derivative between the last two iterations. Infrequently, these differences exceeded $1 \%$ for the flux or $10 \%$ for the flux derivative. In these cases, a different initial model atmosphere was used as input for 30 new ATLAS9 iterations. If the resulting atmosphere was still not converged, additional iterations were computed until the convergence criteria were satisfied.

In very rare cases, it was not possible to achieve these criteria with any number of iterations. These atmospheres were left as is. Most of these atmospheres corresponded to very luminous, warm red giants $\left(6000 \mathrm{~K} \leq T_{\text {eff }} \leq 8000 \mathrm{~K}\right.$ and $0.0 \leq$ $\log g \leq 0.5$ ). These stars lose mass in winds, which violates ATLAS9's assumption of hydrostatic equilibrium. Stars with these atmospheric parameters are exceptionally rare. Therefore, convergence problems in this region of the grid are unlikely to cause problems for real astrophysical applications. The model atmospheres for $\mathrm{M}$ dwarfs ( $T_{\text {eff }} \lesssim 4000 \mathrm{~K}, \log g \lesssim 3.5$ ) also violated the convergence criteria to a small degree in the outer layers, especially at $[\mathrm{Fe} / \mathrm{H}] \lesssim-1$. M dwarf atmospheres have low electron densities and nonblackbody spectral energy distributions, which cause them to be out of statistical equilibrium (Schweitzer 1999). The outer layers affect the strongest 
absorption lines, which are often formed out of LTE, even for warmer and larger stars.

Figure 1 shows some example stellar atmospheres. The parameters for each atmosphere were chosen to lie near a $14 \mathrm{Gyr}$ isochrone from the Victoria-Regina set of isochrones (VandenBerg et al. 2006). For all three models, the microturbulent velocity is $\xi=2 \mathrm{~km} \mathrm{~s}^{-1}$. The parameters plotted are those relevant to the generation of synthetic spectra. From top to bottom, they are the logarithms of the mass depth $\left(\int \rho d x\right)$, temperature $(T)$, gas pressure $\left(P_{\text {gas }}\right)$, electron number density $\left(n_{e}\right)$, and Rosseland mean opacity $\left(\kappa_{R}\right)$. The independent variable is the logarithm of the optical depth. The Castelli \& Kurucz (2003) models for identical stellar parameters are also shown in gray for comparison. Subtle differences between the two sets of atmospheres - most visible in the middle column of Figure 1—occur due to the slight differences in elemental composition and different criteria for convergence. The largest differences occur in the shallowest layers of the atmosphere, which are important only for the formation of the strongest saturated lines.

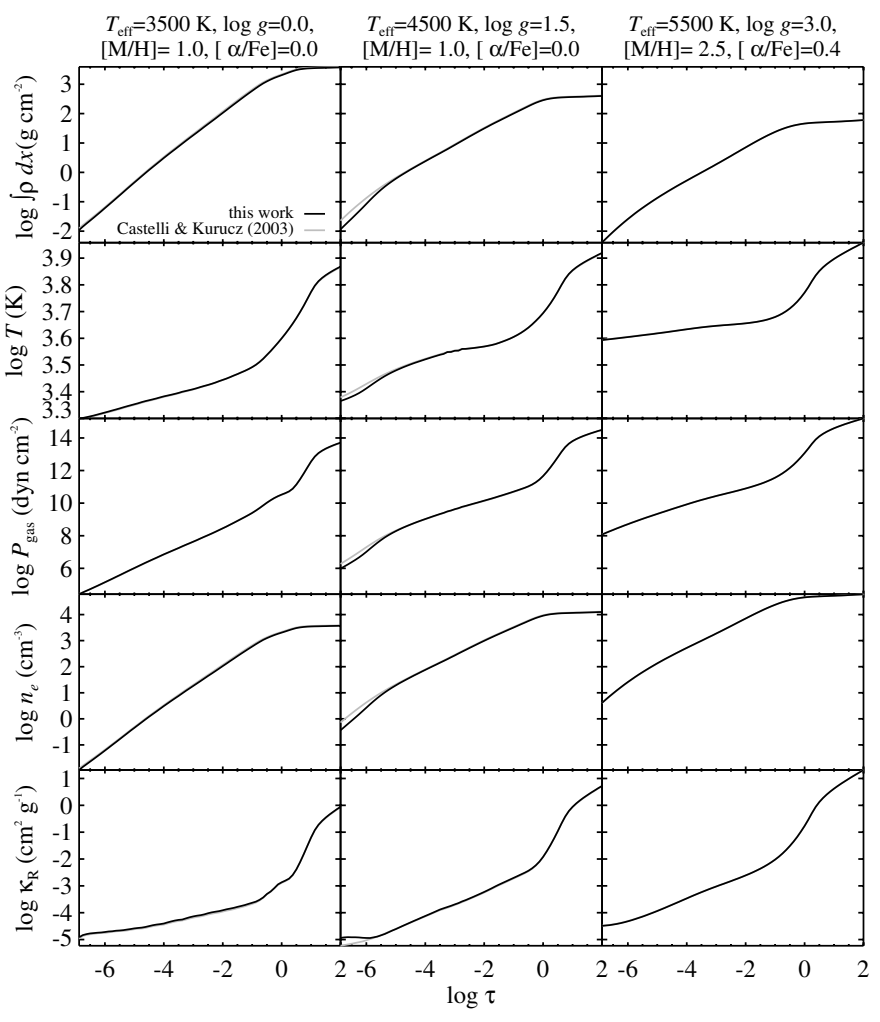

FIG. 1.-ATLAS9 model atmospheres computed here (black) compared with the ATLAS9 model atmospheres with the same parameters computed by Castelli \& Kurucz (2003, gray). The black and gray lines overlap for much of this figure. Each column shows a different star, whose parameters are given at the top of the column. The five rows show the mass depth $\left(\int \rho d x\right)$, temperature $(T)$, gas pressure $\left(P_{\text {gas }}\right)$, electron number density $\left(n_{e}\right)$, and Rosseland mean opacity $\left(\kappa_{R}\right)$ as a function of the logarithm of optical depth $(\log \tau)$.

\section{MOOG SYNTHETIC SPECTRA}

The grid of synthetic spectra was computed with the 2007 version of the plane-parallel, LTE code MOOG (Sneden 1973). The code was modified to run in parallel via OpenMP on 256 processors on the Pleiades cluster. The parallelization was trivial, with different regions of the grid being computed simultaneously. Each spectrum was computed on a single processor. MOOG was also modified to output only the synthetic spectrum into an unformatted binary file. All other output was suppressed to save disk space and file access time. The normalized flux was computed every $0.02 \AA$ from $6300 \AA$ to $9100 \AA$. The units of the spectrum are such that the continuum was unity. The continuum shape was not computed. One spectrum was computed at each grid point.

MOOG requires two data tables as input: a model atmosphere and a line list. The model atmospheres were provided by the ATLAS9 atmospheres described in $\S 2$. However, the spectral grid was five times finer in the $[\mathrm{M} / \mathrm{H}]$ dimension than in the model atmospheres (see Table 1). Therefore, intermediate atmospheres spaced at $0.1 \mathrm{dex}$ in $[\mathrm{M} / \mathrm{H}]$ were generated by linear interpolation of the physical variables (not the logarithms of the physical values).

The microturbulent velocity chosen for each synthetic spectrum is a value appropriate for red giants (eq. 2 of Kirby et al. (2009): $\xi\left(\mathrm{km} \mathrm{s}^{-1}\right)=2.13-0.23 \log g$. The atmosphere used to generate a spectrum was a linear interpolation between the two bracketing values of $\xi$. This linear interpolation was performed in conjunction with linear interpolation in $[\mathrm{M} / \mathrm{H}]$, where necessary.

Kirby et al. (2008) gave the complete line list, but the version used here has been modified according to Kirby et al. (2009). The original line list contained atomic lines from the Vienna Atomic Line Database (VALD; Kupka et al. 1999), molecular lines from Kurucz (1992), and hyperfine atomic lines from Kurucz (1993b). Oscillator strengths were modified to match observed line strengths in Arcturus and the Sun. Kirby et al. (2009) replaced the oscillator strengths $(\log g f)$ for some Fe I lines with the values of Fuhr \& Wiese (2006). For those Fe I lines not shared with Fuhr \& Wiese (2006), 0.13 dex was subtracted from $\log g f$. The only molecules in the line list are $\mathrm{CN}$, $\mathrm{C}_{2}$, and $\mathrm{MgH}$. The opacity for almost every line is considered only within $1 \AA$ of the line's central wavelength. Some lines are considered at every wavelength in the spectrum. These are $\mathrm{H} \alpha$; the hydrogen Paschen series redward of $8370 \AA$; the Ca II triplet at 8498,8542 , and $8662 \AA$; and $\mathrm{Mg}$ I $\lambda 8807$.

Figure 2 shows $100 \AA$ (7900-8000 $\AA$ ) of the synthetic spectra computed from the model atmospheres shown in Figure 1. The full spectral range is 63 times that shown. The abundances used to compute the line strengths are completely consistent with the abundances used to compute the model atmospheres.

Figure 3 shows synthetic spectra compared with spectra observed with the Keck/Deimos medium-resolution spectrograph. Kirby et al. (2010) obtained these spectra. These stars were 


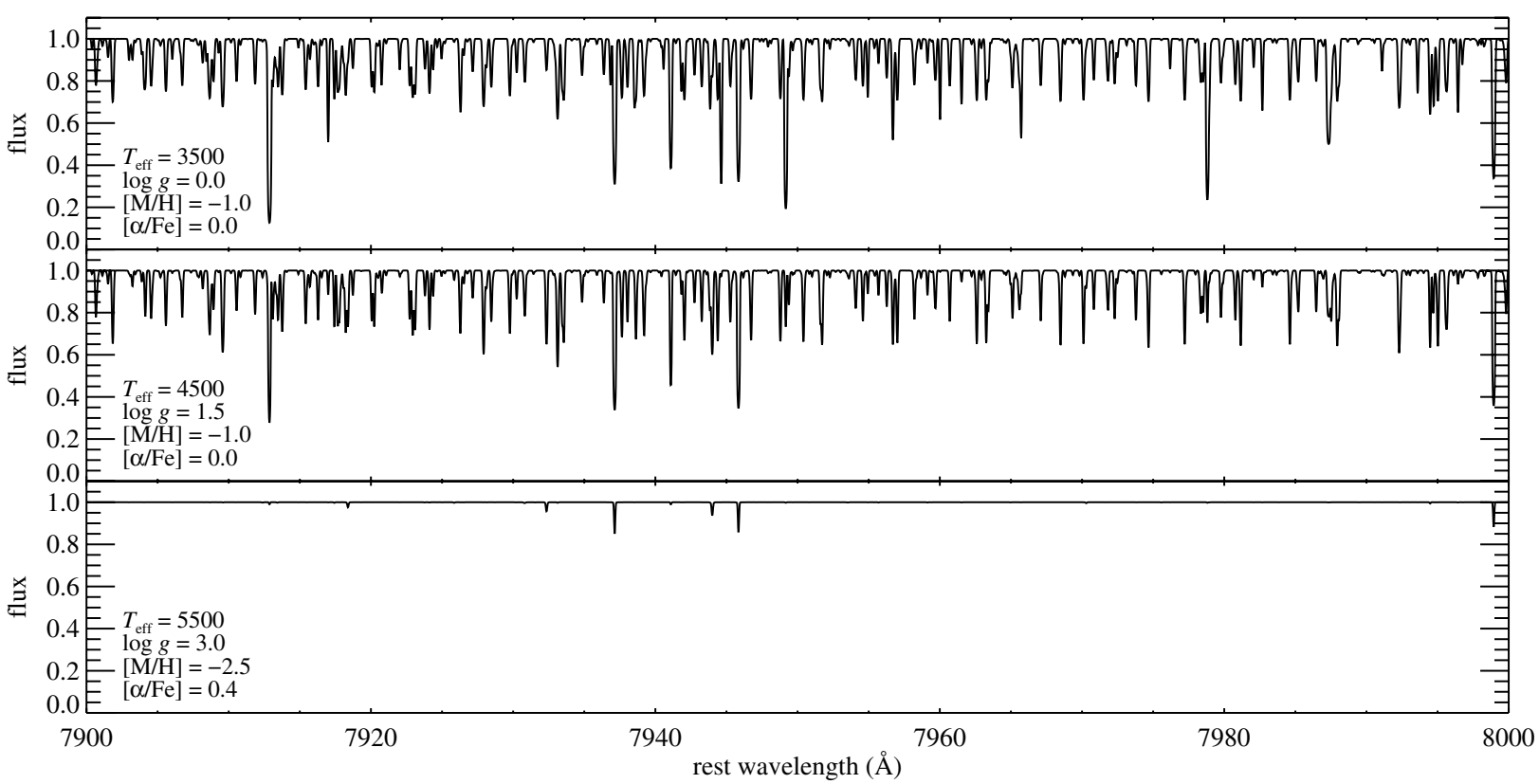

FIG. 2.-Examples of three synthetic spectra computed with MOOG. The stellar parameters and model atmospheres are identical to those shown in Fig.1.

chosen to have atmospheric parameters fairly close to those shown in Figures 1 and 2. The stars are red giants in the globular clusters M5 and M15. The atmospheric parameters for the synthetic spectra are given in the legend at the left of each panel (measured by Kirby et al. 2010). The synthetic spectra were linearly interpolated, pixel by pixel, to reproduce the measured atmospheric parameters. Finally, the synthetic spectral resolution has been degraded to match the Deimos instrumental resolution. In the spectrograph configuration used, profiles of unresolved lines have FWHM $\approx 1.2 \AA$, almost independent of wavelength. Therefore, the resolving power is $R \approx 6200$ at $7400 \AA$ and $R \approx 6700$ at $8700 \AA$.

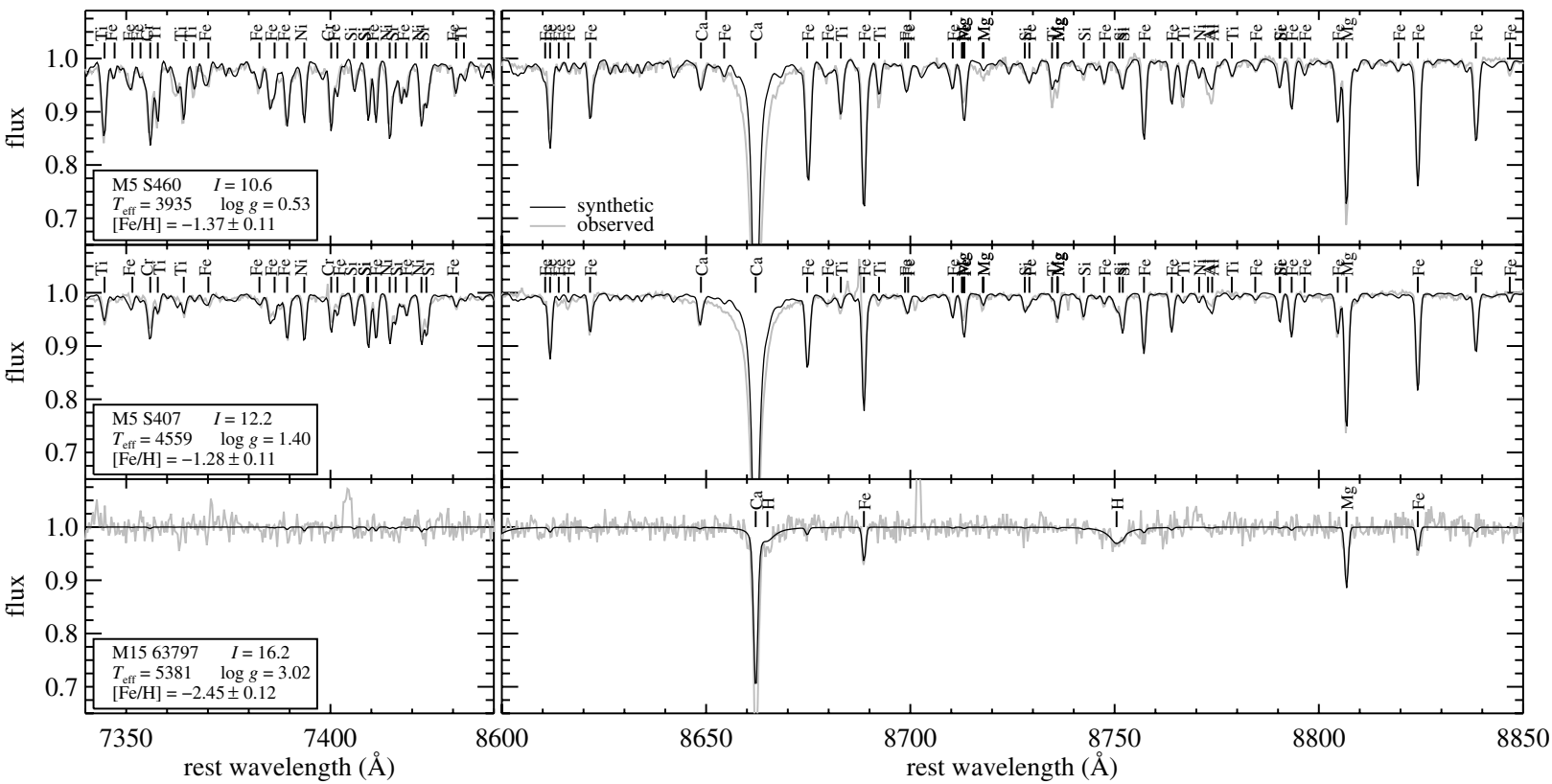

FIG. 3.-Examples of three synthetic spectra (black) compared with spectra observed with Deimos (gray). The synthetic spectra were interpolated in the grid in order to replicate the atmospheric parameters given in the legend at the left of each panel. The interpolated spectra were smoothed to match the resolution of the observed spectra $(R \approx 6500)$. Some of the prominent stellar absorption lines are labeled with the abbreviation of the responsible element. 
The grid of synthetic spectra has several shortcomings, some of which are enumerated here.

1. The only molecules included are $\mathrm{CN}, \mathrm{C}_{2}$, and $\mathrm{MgH}$. The number of $\mathrm{TiO}$ lines makes including them in the spectra computationally prohibitive. Therefore, cool, metal-rich stars are not modeled well in this grid. In reality, their spectra have TiO features that become extremely strong as the temperature decreases and metallicity increases.

2. Strong lines are not modeled well, mostly due to non-LTE effects. For example, Figure 3 clearly shows that the observed wings and core of the $\mathrm{Ca}$ II $\lambda 8662$ line are much stronger than in the synthetic spectrum.

3. Although a relation between microturbulent velocity and surface gravity has been established, not all stars obey this trend. In fact, the relation that Kirby et al. (2009) adopted was based only on red giants. The relation is not appropriate for most subgiants and dwarfs. The user is strongly cautioned against using any synthetic spectra with $\log g>3.5$ without verifying that the microturbulent velocity is appropriate for the specific star under consideration.

\section{ONLINE DATA ACCESS}

The grids of model atmospheres and synthetic spectra may be obtained at the VizieR online catalog service (Ochsenbein et al. 2000). ${ }^{6}$ The user has two options to access the catalog. First, the catalog may be accessed either through the VizieR online interface, which includes an option to plot the synthetic spectra in a web browser. Second, one or both of the grids may be downloaded via FTP or HTTP.

The author thanks Joel Primack for sharing his time on the Pleiades Astrophysics Computing Cluster at the University of California, Santa Cruz, supported by a grant from the National Science Foundation Major Research Instrumentation program; Judy Cohen for sharing a list of helpful references; François Ochsenbein for his help in preparing the data for online access via VizieR; F. Castelli for her helpful comments; and R. Kurucz, the referee, whose suggestions improved this article. Support for this work was provided by NASA through Hubble Fellowship grant 51256.01 awarded to E. N. K. by the Space Telescope Science Institute, which is operated by the Association of Universities for Research in Astronomy, Inc., for NASA, under contract NAS 5-26555. This research has made use of the VizieR catalog access tool, CDS, Strasbourg, France.

\footnotetext{
${ }^{6}$ See http://vizier.u-strasbg.fr/viz-bin/VizieR?-source=VI/134.
}

\section{REFERENCES}

$\rightarrow$ Anders, E., \& Grevesse, N. 1989, Geochim. Cosmochim. Acta, 53, 197 Castelli, F. 2005, Mem. Soc. Astron. Ital. Supp., 8, 34

Castelli, F., \& Kurucz, R. L. 2003, in IAU Symp. 210, Modelling of Stellar Atmospheres (Cambridge: Cambridge Univ. Press), 20P

$\rightarrow$ Coelho, P., Barbuy, B., Meléndez, J., Schiavon, R. P., \& Castilho, B. V. 2005, A\&A, 443, 735

$\rightarrow$ Frebel, A., Simon, J. D., Geha, M., \& Willman, B. 2010, ApJ, 708, 560

$\rightarrow$ Fuhr, J. R., \& Wiese, W. L. 2006, J. Phys. Chem. Ref. Data, 35, 1669 Grevesse, N., \& Sauval, A. J. 1998, Space Sci. Rev., 85, 161

$\rightarrow$ Kirby, E. N., Guhathakurta, P., Bolte, M., Sneden, C., \& Geha, M. C. 2009, ApJ, 705, 328

$\rightarrow$ Kirby, E. N., Guhathakurta, P., \& Sneden, C. 2008, ApJ, 682, 1217

$\rightarrow$ Kirby, E. N., et al. 2010, ApJS, 191, 352

$\rightarrow$ Kupka, F., Piskunov, N., Ryabchikova, T. A., Stempels, H. C., \& Weiss, W. W. 1999, A\&AS, 138, 119

Kurucz, R. L. 1970, ATLAS: A Computer Program for Calculating Model. Stellar Atmospheres (SAO Spec. Rep.309; Cambridge: $\mathrm{SAO})$

- 1990, in Stellar Atmospheres: Beyond Classical Models, ed. L. Crivellari, I. Hubený, \& D. G. Hammer (NATO ASI Ser. C, 341; Dordrecht: Kluwer), 441

- 1992, Rev. Mex. AA, 23, 45 1993a, Kurucz CD-ROM 13, ATLAS9 Stellar Atmosphere Programs and $2 \mathrm{~km} / \mathrm{s}$ Grid (Cambridge: SAO)

$\rightarrow$ 1993b, Phys. Scr., T47, 110

$\rightarrow$ Letarte, B., et al. 2010, A\&A, 523, A17

$\rightarrow$ Mihalas, D. 1965, ApJS, 9, 321

$\rightarrow$ Munari, U., Sordo, R., Castelli, F., \& Zwitter, T. 2005, A\&A, 442, 1127

$\rightarrow$ Ochsenbein, F., Bauer, P., \& Marcout, J. 2000, A\&AS, 143, 23

$\rightarrow$ Palacios, A., Gebran, M., Josselin, E., Martins, F., Plez, B., Belmas, M., \& Lèbre, A. 2010, A\&A, 516, A13

$\rightarrow$ Partridge, H., \& Schwenke, D. W. 1997, J. Chem. Phys., 106, 4618 Sbordone, L. 2005, Mem. Soc. Astron. Ital. Supp., 8, 61

Sbordone, L., Bonifacio, P., Castelli, F., \& Kurucz, R. L. 2004, Mem. Soc. Astron. Ital. Supp., 5, 93

Schweitzer, A. 1999, Ph.D. thesis, Landessternwarte Heidelberg/ Königstuhl (Germany)

$\rightarrow$ Schwenke, D. W. 1998, Faraday Discussions, 109, 321

Sneden, C. A. 1973, Ph.D. thesis, University of Texas at Austin

$\rightarrow$ Strom, S. E., \& Avrett, E. H. 1965, ApJS, 12, 1

$\rightarrow$ Strom, S. E., \& Kurucz, R. L. 1966, AJ, 71, 181

$\rightarrow$ VandenBerg, D. A., Bergbusch, P. A., \& Dowler, P. D. 2006, ApJS, 162,375 